\title{
TECNOLOGIAS EDUCATIVAS NA PROMOČ̃̃O DA SAÚDE SEXUAL DO ADOLESCENTE COM DEFICIẾNCIA VISUAL
}

\author{
Amanda Serra Souza', Ana Paula Rodrigues Silva', Carolina Magalhães dos Santos ${ }^{2}$ \& Aline \\ Siqueira de Azevedo 3
}

SOUZA, A.S.; SILVA, A.P.R.; SANTOS, C.M. \& AZEVEDO, A.S. Tecnologias educativas na promoção da saúde sexual do adolescente com deficiência visual. Perspectivas Online: Biológicas \& Saúde. v. 8, n 28, p.81-91, 2018.

\section{RESUMO}

As tecnologias educativas e inclusivas compõem duas realidades presentes na escola. Todavia, existe um longo percurso para que o uso das tecnologias da informação e da comunicação promova as tão desejadas práticas inclusivas em contextos de educação formal e não-formal. A relevância sobre a temática de orientação ao adolescente com deficiência visual quanto à prevenção e promoção de Infecções Sexualmente Transmissíveis é indiscutível. Utilizar as tecnologias educativas de forma fundamentada e planificada pode gerar atitudes positivas frente à diversidade $\mathrm{e}$ proporcionar ganhos à nível acadêmico e social, do desenvolvimento de um sentido de comunidade e das práticas nãodiscriminatórias, que são as componentes da filosofia inclusiva. $\mathrm{O}$ presente estudo investigou a eficácia da utilização das tecnologias educativas na promoção de saúde sexual do adolescente com deficiência visual e conheceu as suas percepções acerca da sexualidade. É apresentada uma abordagem qualitativa, através do método descritivoexploratório; utilizando um formulário de perguntas semiestruturadas. Os sujeitos envolvidos nessa pesquisa foram usuários do Educandário São José dos Operários, situado no município de Campos dos Goytacazes, com idade entre 15 anos e 18 anos. Conhecer as percepções dos adolescentes com deficiência visual acerca de sua sexualidade oportunizou verificar que esses adolescentes carecem de informações e conhecimentos com relação a diversas questões que envolvem a sexualidade. Sendo assim, podemos concluir que a educação em saúde e a educação inclusiva caminham lado a lado na proposta da acessibilidade e empoderamento do deficiente visual para o cuidado de saúde, bem como no âmbito da saúde sexual e reprodutiva para a prevenção de infecções sexualmente transmissíveis.

Palavras-chave: Educação em saúde, Enfermagem, Sexualidade. 


\begin{abstract}
Educational and inclusive technologies make up two realities present in the school. However, there is a long way to use information and communication technologies as a way of making use of formal and nonformal education contexts. The evaluation about the subject of teenager with visual deficiency to the prevention and research of Sexually Transmitted Infections is indisputable. Use educational technologies in a well-grounded and planned way to generate positive attitudes toward academic and social differences and modes of learning, developing a sense of community and non-discriminatory practices, which are components of inclusive philosophy. The present study investigated the use of educational technologies in the promotion of adolescent sexual health with

perceptions about sexuality. It is a qualitative approach, through the descriptive-exploratory method; Using a semi-structured question form. The students are looking for users of Educandário São José dos Operários, located in the municipality of Campos dos Goytacazes, aged between 15 years and 18 years. Knowing the perceptions of adolescents with the visual about their sexuality has facilitated the selection of adolescents from careers and information regarding the different issues involving a sexuality. Thus, we can conclude that health education and inclusive education is a theme of the accessibility and empowerment of the visually impaired for health care, as well as sexual and reproductive health for the prevention of sexually transmitted infections.
\end{abstract} visual impairment and was aware of their

Keywords: Health education, Nursing, Sexuality.

1 Institutos Superiores de Ensino do CENSA -ISECENSA - Aluno (a) Voluntário (a) de Iniciação Científica PROVIC - Rua Salvador Correa, 139, Centro, Campos dos Goytacazes, RJ, CEP: 28035-310, Brasil.

2 Pesquisadora do Laboratório de Estudos em Saúde Pública - LAESP/ISECENSA.

${ }^{3}$ Pesquisadora do Núcleo de Pesquisa de Fundamentos do Cuidado de Enfermagem - NUPEFE/ISECENSA.

(*)e-mail: enfsiqueira@gmail.com

Data de recebimento: 23/11/2018 Aceito para publicação: 19/12/2018 


\section{INTRODUÇÃO}

As tecnologias educativas e a inclusão constituem duas realidades presentes na sociedade atual e na sociedade do futuro. Todavia, há um longo percurso para que o uso das tecnologias da informação e da comunicação possibilite promover as tão desejadas práticas inclusivas em contextos de educação. Utilizando de forma fundamentada e planificada às tecnologias educativas podem promover atitudes positivas diante à diversidade e promover ganhos ao nível acadêmico e social, do desenvolvimento de um sentido de comunidade e das práticas não discriminatórias, que são as componentes da filosofia inclusiva (CARVALHO, 2017)

Tais tecnologias aparecem como prioritárias para fazer frente o principal objetivo educacional: "garantir uma permanente ação formativa orientada para favorecer o desenvolvimento global da personalidade, o progresso social e a democratização da sociedade", como especificado no $2^{\circ}$ parágrafo do artigo $1^{\circ}$ da Lei no 49/2005 de 30 de Agosto da Lei Bases do Sistema Educativo.

No aspecto epidemiológico contamos com uma vasta literatura a respeito das chamadas "deficiências", que propõe novas práticas e novos conceitos: enquanto alguns autores defendem a criação de possibilidades de acesso, inclusão, cidadania e ética, outros, por sua vez, denunciam o leixamento e a exclusão. Fala-se, sobretudo no advento das novas tecnologias e o seu potencial equalizador como uma das referências principais à inclusão (ESTABEL; MORO; SANTAROSA, 2006).

Deve-se observar o que é dito e o que é subentendido para compreender todo o processo da inclusão/exclusão manifestado e executado nos dias de hoje. Amparados na teoria histórico cultural nota-se questões específicas, tais como: possibilidades e perspectivas presentes nos instrumentos e equipamentos, tendo como finalidade detectar como as novas tecnologias contribuem para a melhoria de vida dos deficientes visuais (SIMÃO, 2010).

O indivíduo com deficiência visual tem restrito seu acesso a informações sobre a educação para a saúde, visto que essas, em sua maioria, utilizam a visão como estratégia de comunicação. Dado esse contexto, deve-se assumir a responsabilidade junto à sociedade enquanto cuidadores, pesquisadores e educadores para a saúde, contribuindo para o desenvolvimento e integração social dos deficientes visuais (BRASIL, 2009).

No Brasil a educação sexual é quase restrita a estímulos visuais, há escassez em programas de educação sexual adaptados ao deficiente visual. Já que esses indivíduos não podem aprender por imitação visual, o ideal seria que todos os movimentos lhes fossem demonstrados e com eles juntamente realizados, explorando-se, dessa forma, os sentidos remanescentes do deficiente visual, tato e audição, de modo a contribuir para o melhor entendimento das informações (COSTA; SILVA, 2017).

A educação em saúde não é uma hipótese abstrata, é uma realidade que responde às necessidades de saúde e à possibilidade objetiva de adquirir comportamentos positivos. Acredita-se que as pessoas, mesmo as com necessidades especiais e, nesse caso, os deficientes visuais, devem poder ter suas próprias decisões e conhecimentos sobre sua saúde, exercendo assim seus direitos e deveres para o pleno exercício de sua cidadania (RIBEIRO; et al., 2018). 
Refletindo sobre o papel do educador em saúde, visualiza-se, no contexto do deficiente visual, a possibilidade de se diminuir os conflitos e inquietações que acompanham esses indivíduos e, dessa forma, auxiliá-los no exercício de uma sexualidade livre de preconceitos e mal-entendidos (BRASIL, 2009).

Diante de todo exposto, o presente trabalho tem como objetivo investigar a eficácia da utilização das tecnologias educativas na promoção de saúde sexual do adolescente com deficiência visual e conhecer as percepções do deficiente visual acerca de sua sexualidade.

\section{METODOLOGIA}

O estudo trata de uma pesquisa descritiva-exploratória com abordagem qualitativa, realizado no Educandário São José dos Operários no município de Campos dos Goytacazes/RJ. O Educandário é uma instituição filantrópica, que visa a promoção da inclusão social de pessoas com deficiência visual.

A amostra do estudo foi composta por quatro adolescentes com deficiência visual que frequentavam o Educandário São José dos Operários tendo como critério de inclusão: adolescentes com deficiência visual, independente de ser essa congênita ou adquirida e cujos pais e/ou responsáveis autorizassem a participação. E, como fator de exclusão: adolescentes com deficiência neurológica grave.

A primeira etapa ocorreu em outubro de 2017, foi realizada a caracterização dos sujeitos e seus conhecimentos acerca da sexualidade por meio de um formulário com perguntas abertas e fechadas. Posteriormente, foi marcado um segundo encontro, em abril de 2018, a fim de obter o conhecimento desses sujeitos sobre Infecções Sexualmente Transmissíveis (IST's) através da aplicação do formulário pré-teste. Logo após, foram utilizados manequins, como tecnologia educativa, que simulavam aspectos, texturas e odores das patologias além de esclarecer dúvidas sobre ISTs.

A terceira etapa da pesquisa ocorreu em maio de 2018 com o retorno a instituição para aplicação do formulário pós-teste, a fim de avaliar a eficácia do uso das tecnologias educativas na promoção da saúde sexual. Os encontram ocorreram em datas estipuladas, conforme a disponibilidade dos sujeitos, em espaço reservado na instituição, de forma individual, respeitando a privacidade de cada um deles.

As informações obtidas foram submetidas à técnica de analise de conteúdo. A transcrição das falas constituíram as categorias identificadas após a realização das etapas.

O presente estudo foi submetido e aprovado pelo Comitê de Ética em Pesquisa com Seres Humanos do ISECENSA (Campos dos Goytacazes, RJ), sendo elaborado um Termo de Consentimento Livre e Esclarecido entregue aos pais pelos adolescentes. Além da autorização dos pais, leu-se o termo de consentimento, em voz alta, na presença dos entrevistados para que os mesmos concordassem e assinassem. Para a manutenção do anonimato dos sujeitos os mesmos foram denominados com nomes de pedras preciosas. 


\section{RESULTADOS E DISCUSSÃO}

O Educandário São José dos Operários é composto por crianças, adolescentes, adultos e idosos com deficiência sexual parcial e/ou total. A pesquisa foi constituída por 04 adolescentes com deficiência visual.

Em relação às características sociodemográficas da amostra, observamos uma faixa etária entre 15 e 18 anos. Quanto ao gênero, 02 eram do sexo feminino e 02 do sexo masculino. Quanto ao nível de deficiência visual, os 03 possuem perda parcial. Relacionado ao grau de instrução, 02 cursavam o sétimo ano e 01 o quinto ano do ensino fundamental 2. Verificamos que o tempo médio dos adolescentes na instituição é de meio período, onde há uma complementação de atividades específicas para deficientes visuais.

\subsection{A percepção do adolescente visual quanto a sua sexualidade:}

Durante o processo de desenvolvimento do corpo ocorrem mudanças que os adolescentes com deficiência visual, apesar de não poderem enxergar as mudanças em seus corpos, percebem que estão crescendo e que mudanças estão acontecendo no seu corpo. Notam estruturas que antes não possuíam como os seios para as meninas ou a barba para os meninos.

Tal evidência é concebível, pois as modificações fisiológicas que caracterizam esse processo proporcionam a experiência de uma série de eventos psicológicos que terminam no que se denomina aquisição de identidade sexual.

De acordo com Freitas e Dias (2010) sexualidade é o desejo de contato, o amor, carinho, atração, beijo, calor e também é identificado através do pensamento, fantasias, desejos, opiniões, atitudes, valores, comportamentos, práticas e nos relacionamentos.

Quando os entrevistados foram questionados sobre sexualidade obtivemos os seguintes fragmentos transcritos abaixo:

"De relação. Tem que ter muito cuidado com essas coisas”

(Ágata)

"Não sei. Tenho vergonha."

(Turquesa)

As narrativas demonstram, em sua totalidade, que os deficientes visuais demonstram falta de conhecimento e informações erradas com relação à sexualidade. Fato agravado pela carência de materiais informativos relacionados a essa temática, adaptados ao deficiente visual, dificultando o acesso dos mesmos a informações precisas.

Os adolescentes com deficiência visual encaram diversas dificuldades ao expressar sua sexualidade, tanto pelo preconceito quanto pela carência de informações sobre sexualidade. Trata-se 
de uma comunidade que sofre de exclusão social e que necessita de recursos diferentes e criativos para que o processo de aprendizagem seja realizado e compreendido com eficácia (COZAC; PEREIRA; CASTRO, 2016).

Em determinado momento da entrevista fomos surpreendidas com a pergunta:

"Se o menino passar a mão lá, corre o risco de engravidar?"

(Ágata)

“Como eu faço para saber se estou grávida?”

(Ágata)

"Como se conta para a família caso esteja grávida?"

(Ágata)

"O que é menstruação?”

(Turquesa)

A adolescência é classificada como um estágio de descobertas devido as transformações físicas e biológicas junto com as questões psicológicas e sociais, da própria fase. E é nesta fase que o interesse sobre a sexualidade surge cabendo aos responsáveis uma breve conversa com o jovem e uma abordagem mais completa dos profissionais das escolas e da equipe de saúde, resultando na necessidade de esclarecimentos sobre saúde e sexualidade, tendo como finalidade visar a proteção e prevenção de agravos a saúde e a vida (MELO, 2017).

Gomes e colaboradores (2010) relatam que é de extrema importância um diálogo claro e objetivo com o deficiente visual sobre sexualidade e as mudanças físicas e biológicas que ocorrem com seu corpo na transição da fase infantil para a fase da adolescência, para que seja inserido no contexto social e cultural.

Alguns responsáveis de crianças com deficiência visual segundo da Silva e colaboradores (2017) costumam ter o comportamento superprotetor e acabam não fornecendo informações suficientes sobre sexualidade e as mudanças normais que o corpo humano passa, às vezes por medo ou por não saber como passar tais informações para eles devido à falta de material.

Segundo Cezario e Pagliuca (2007), a literatura em braile é escassa quando se trata de educação em saúde, principalmente sexualidade, os recursos disponíveis destinam-se a pessoas videntes, sendo as informações transmitidas de forma bastante superficial e não atendendo adequadamente às necessidades de um deficiente visual.

O Artigo 25 da Convenção sobre os Direitos da Pessoa com Deficiência aborda o direito de acesso aos programas de atenção à saúde, inclusive à saúde sexual e reprodutiva. No documento, a sexualidade é considerada não somente como instinto sexual nem atividades que dependem do funcionamento do aparelho genital, mas por grande plasticidade e relação com a história pessoal de cada indivíduo. 
3.3 Tecnologias educativas na promoção da saúde sexual do adolescente com deficiência visual.

As tecnologias educativas e a inclusão constituem duas realidades presentes na sociedade atual e na sociedade do futuro. Todavia, há um longo percurso para que o uso das tecnologias da informação e da comunicação possibilite promover as tão desejadas práticas inclusivas em contextos de educação.

Utilizando de forma fundamentada e planificada às tecnologias educativas podem promover atitudes positivas diante à diversidade e promover ganhos ao nível acadêmico e social, do desenvolvimento de um sentido de comunidade e das práticas não discriminatórias, que são as componentes da filosofia inclusiva (SANTOS; et al., 2018).

Nesse sentido, foi questionado aos entrevistados acerca de materiais que eles tem acesso para sanar dúvidas sobre sexualidade e IST's e os resultados apontaram que $100 \%$ dos adolescentes não tem acesso a esse tipo de material. Mediante a esta informação justifica-se a seguinte transcrição:

"Não tem nenhum material e não tenho interesse em procurar."

(Topázio)

As tecnologias leves apresentam-se, atualmente como destaque na educação em saúde, pois garante um aprendizado que possibilita a democratização da sociedade, o desenvolvimento global da personalidade e o progresso social, como citado na Lei 49/2005 de 30 de Agosto da Lei Bases do Sistema Educativo.

As informações que são divulgadas através dos meios de comunicação como televisão, jornais, revistas, cartazes e folders, distribuídos em diversos locais, não atingem de maneira eficaz o deficiente visual. Faltam programas de educação sexual que sejam adaptados ao deficiente visual, com informações escritas em braile e que privilegiem os demais sentidos, posto que a visão não é o único meio de que se dispõe para divulgar e apreender informações.

Durante a entrevista os sujeitos foram questionados quanto as principais patologias das ISTs, forma de contágio, sinais e sintomas e prevenção. Nesse momento a entrevista foi dividida em dois momentos, pré-teste e pós-teste.

Após a fase pré-teste, as pesquisadoras deram início a fase pós-teste onde foram utilizados materiais específicos como preservativos masculinos, manequins de genitália masculina e feminina, para simular sinais e sintomas das ISTs, como por exemplo: herpes, sífilis, gonorreia, como observado na figura 1. 

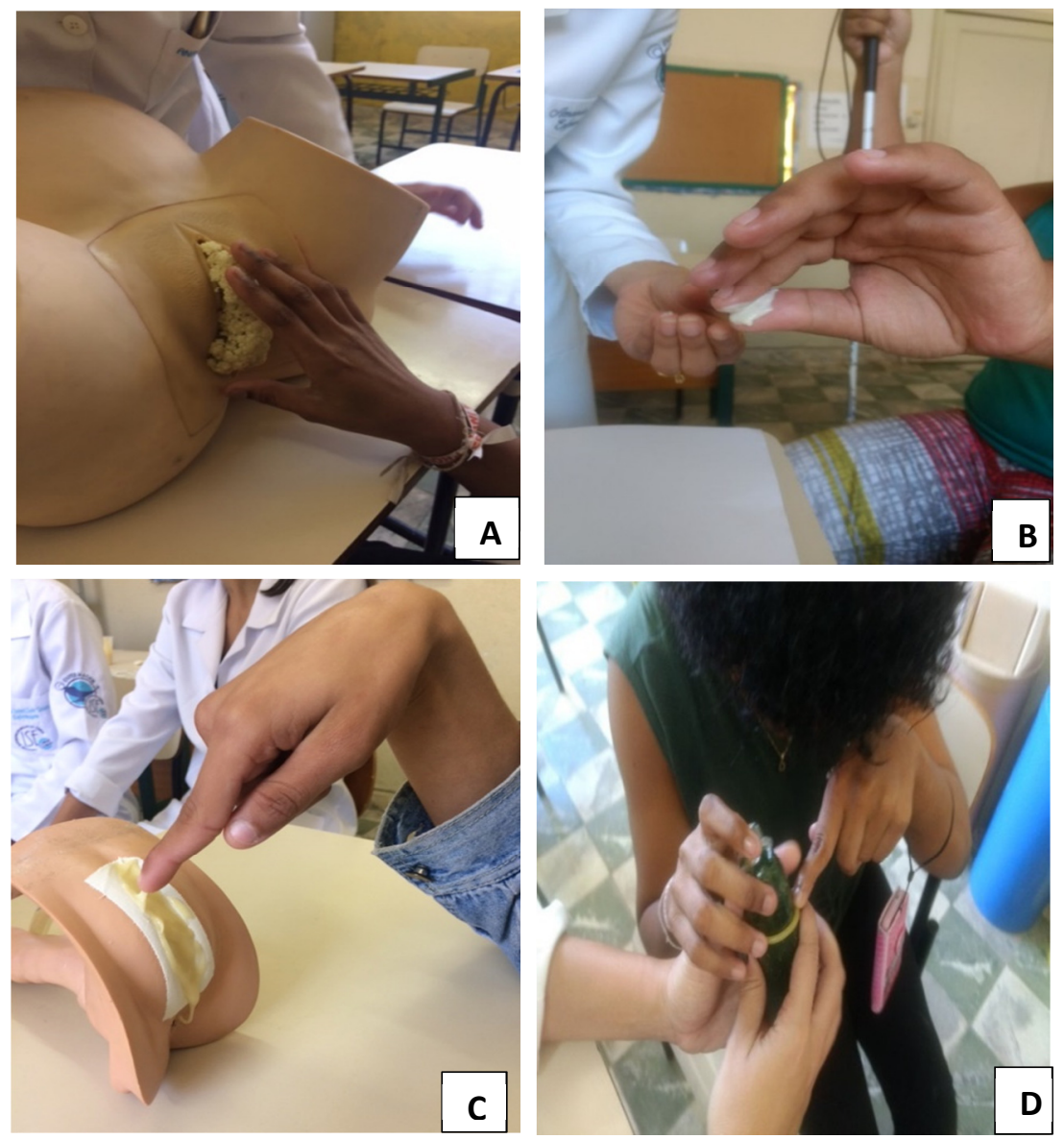

Figura 1 A-D: Utilização de Tecnologias Educativas na promoção da educação em saúde para adolescentes com deficiência visual assistidos no Educandário São José dos Operários em Campos dos Goytacazes/RJ.

Na fig 1A, observa-se a utilização de um manequim de pelve feminina com couve flor representando lesões relacionadas à sífilis. Nesse momento os sujeitos tocavam na pelve para sentir a textura.

Nas figs 1B e C, observa-se a utilização de um manequim, de uma vagina, com uma secreção feita de leite condensado, trigo e gotas de óleo de peixe, para representar a gonorreia. Neste momento os sujeitos tocaram a peça para sentir a textura e levaram ao nariz para sentir o odor que a patologia pode apresentar.

Na fig 1D, observa-se a utilização de um penino para ajudar na compreensão da colocação de um preservativo masculino. Neste momento, foi ensinado a melhor formar de abrir o pacote de preservativo e auxiliou-se na colocação do mesmo.

Também foi utilizada durante o período de palestras um manequim, de uma vagina, com pedaços de plástico bolha contendo uma pequena quantidade de água dentro, para a representação da herpes. Neste momento os sujeitos tocaram para sentir a textura das bolhas para melhor 
elucidação da patologia.

A educação em saúde é definida como “[...] um processo planificado e sistemático de comunicação e de ensino-aprendizagem orientado a facilitar a aquisição, escolha e manutenção das práticas saudáveis e dificultar as práticas de risco" (BRASIL, 2009).

Os problemas que atingem os deficientes visuais, de qualquer faixa etária, como gestação indesejada, aborto, Infecções Sexualmente Transmissíveis, HIV/AIDS são alvo de preocupação entre formuladores de políticas e educadores de um modo geral. O preparo dos jovens para o exercício saudável da sexualidade requer intervenção planejada por parte dos profissionais da saúde e da educação que estejam fundamentadas em uma concepção de adolescência, sexualidade, educação sexual de modo a atender a integralidade humana, em que a sexualidade seja compreendida como uma das expressões que envolvem afetos, sentidos, desejos, comunicação e criação (CEZARIO; PAGLICA, 2007).

\section{CONCLUSÃO}

Inegavelmente a falta de visão impõe restrições na vida do indivíduo. Entretanto, por meios de adaptações na utilização dos demais sentidos, é possível desenvolver inúmeros recursos que permitam estas pessoas conhecer o mundo em que vivem.

Desta forma, a criação e validação de materiais educativos se fazem necessárias ao se inserir na proposta da acessibilidade, no empoderamento do deficiente visual para o cuidado de saúde, bem como no âmbito da saúde sexual e reprodutiva para a prevenção de doenças sexualmente transmissíveis.

Os adolescentes com deficiência visual devem ser incentivados a uma prática sexual saudável através de uma educação em saúde sexual, incluindo métodos contraceptivos e o uso de preservativo. Para tanto torna-se necessário desenvolver recursos que facilitem a comunicação com o deficiente visual para que os mesmos se tornem mais independentes no seu autocuidado.

É indiscutível a relevância da temática de orientação dessa população quanto à prevenção das ISTs. Como evidenciado em estudo, tal como a população geral, os deficientes visuais também precisam de educação, valendo-se de inovações e avanços tecnológicos. Como asseguram pesquisadores, o uso de meios lúdicos que promovam a aprendizagem e despertem interesse, pode induzir a mudança de comportamento.

Conhecer as percepções dos adolescentes com deficiência visual acerca de sua sexualidade oportunizou verificar que esses adolescentes carecem de informações e conhecimentos com relação a diversas questões que envolvem a sexualidade.

Sendo assim, podemos concluir que a educação em saúde e a educação inclusiva caminham lado a lado na proposta da acessibilidade e empoderamento do deficiente visual para o cuidado de saúde, bem como no âmbito da saúde sexual e reprodutiva para a prevenção de infecções sexualmente transmissíveis. 


\section{REFERENCIAS}

BRASIL. Decreto $n^{\circ}$ 6.949, de 25 de agosto de 2009. Promulga a convenção internacional sobre os direitos das pessoas com deficiência. Diário Oficial [da] República Federativa do Brasil, Brasília, DF, 26 ago. 2009. Seção 1, p. 3 [acesso em 2016 Jan 02]. Disponível em: <http://www.planalto.gov.br/ccivil_03/_ato2007-2010/2009/decreto/d6949.htm>. Acesso em: 09 Jul. 2018.

BRASIL. Ministério da Saúde. Secretaria de Atenção à Saúde. Departamento de Ações programáticas Estratégicas. Área técnica saúde da pessoa com deficiência. Direitos sexuais e reprodutivos na integralidade das ações à saúde de pessoas com deficiência. Brasília,2009. Disponível em: <http://bvsms.saude.gov.br/bvs/publicacoes/saude_sexual_reprodutiva_homens_cuidado.pdf>. Acesso em: 20 Mar. 2017.

CARVALHO, A. S. Educação inclusiva: práticas docentes frente à deficiência auditiva. Faculdade Anhanguera, Guarulhos, 2017. Disponível em: $<$ http://repositorio.pgsskroton.com.br/bitstream/123456789/16493/1/ANANDA\%20DOS\%20SAN TOS\%20CARVALHO.pdf >. Acesso em: 09 Jul. 2018.

CEZARIO, K. G.; PAGLIUCA, L. M. F. Tecnologia para cegos e prevenção às drogas. Esc. Ana Nery, v.11, $\mathrm{n}^{\mathrm{o}} \quad 4, \quad$ p. 677- 681, 2007. Disponível em: < http://www.scielo.br/pdf/ean/v11n4/v11n4a19.pdf>. Acesso em: 06 Mar. 2017.

COSTA, Z. L. S.; SILVA, D. R. Q. Educação sexual no Brasil: uma revisão bibliográfica do período de 2005 a 2015. SEFIC 2015, 2017. Disponível em: $<$ https://anais.unilasalle.edu.br/index.php/sefic2015/article/viewFile/267/205>. Acesso em: 09 Jul. 2017.

COZAC, M. C., PEREIRA, A.R., CASTRO, S.S. Concepção de sexualidade entre pessoas com deficiência visual. Cad. Edu. Saúde e Fis. v.3, n. 6, 2016. Disponível em: <https://www.researchgate.net/publication/325157460_CONCEPCAO_DE_SEXUALIDADE_EN TRE_PESSOAS_COM_DEFICIENCIA_VISUAL>. Acesso em: 08 Jul. 2018.

DA SILVA, Núbia Xavier et al. Superproteção dos pais: Entraves e Influências na Independência de Alunos Deficientes Visuais no Espaço Escolar. In: VIII SEMPP \& I SINTEC. $2017 . \quad$ Disponível em:

http://www.eventos.unir.br/index.php/viii_spgp_i_sipitt/semppsintec/paper/view/562/0>. Acesso em: 12 Dez 2018.

ESTABEL, L. B.; MORO, E. L. S. ; SATAROSA, L. M. C. A inclusão social e digital de pessoas com limitação visual e o uso das tecnologias de informação e comunicação na produção de páginas para internet. Cienc. Inform., v. 35, $\mathrm{n}^{\circ}$ 1, p. 94-101, 2006. Disponível em: s http://www.scielo.br/pdf/ci/v35n1/v35n1a10.pdf>. Acesso em: 09 Nov. 2017.

FREITAS, K.R., DIAS, S.M. Percepções de adolescentes sobre sua sexualidade. Texto \& Contexto Enfermagem [em linea], v.2, p.19, abr-jun, 2010. Disponível em: $<$ http://www.redalyc.org/html/714/71416097017/>. Acesso em: 08 Jul. 2018.

MELO, M.C.P. Sexualidade na adolescência: entrelaçando atitudes, posturas e estratégias em sala de aula com apoio da Estratégia Saúde da Família. Tese (Doutorado) - Universidade Federal do Rio Grande do Sul, Porto Alegre, 2017. Disponível em: 
<http://www.bibliotecadigital.ufrgs.br/da.php?nrb=001048778\&loc=2017\&l=bf86c43728d29732>. Acesso em 08 Jul. 2018.

RIBEIRO, D. K., et al. Experiência de estudantes de enfermagem em um projeto de educação em saúde e sexualidade na escola. Revista Guará, v. 6, n. 10, 2018. Disponível em: $<$ http://periodicos.ufes.br/guara/article/view/15624/13680>. Acesso em: 09 Jul. 2018.

SANTOS, A.S.; et al. Tecnologia educacional baseada em nola pender: promoção da saúde do adolescente. Rev. Enferm. UFPE online, Recife, v.12, n.2, p. 582-588, 2018. Disponível em: $<$ https://periodicos.ufpe.br/revistas/revistaenfermagem/article/view/22609/27896>. Acesso em: 08 Jul. 2018.

SIMÃO, V. S. S. Recursos e estratégias para o ensino de pessoas cegueira e baixa visão. Palestra no Seminário em Florianópolis no dia 01.07.2010. Disponível em: <http://www.pmf.sc.gov.br/arquivos/arquivos/pdf/05_07_2010_13.47.58.f1e04ed09e4fcd73d246a0 8cccf694a6.pdf>. Acesso em: 25 Mar. 2017.

GOMES, Nilvia et al. A diversidade na educação inclusiva e a sexualidade da pessoa com deficiência visual. InterSciencePlace, v. 12, n. 3, 2017. Disponível em: <http://interscienceplace.org/isp/index.php/isp/article/view/672/412>. Acesso em: 12 Dez. 2018. 